\title{
Low-Temperature in Situ Large Strain Plasticity of Ceramic SiC Nanowires and Its Atomic-Scale Mechanism
}

\author{
X. D. Han, ${ }^{\dagger}$ Y. F. Zhang, ${ }^{\dagger}$ K. Zheng, ${ }^{\dagger}$ X. N. Zhang, ${ }^{\dagger}$ Z. Zhang, ${ }^{*}{ }^{\dagger}$ Y. J. Hao, ${ }^{\ddagger}$ \\ X. Y. Guo, ${ }^{\ddagger}$ J. Yuan,,$^{\S}$ and Z. L. Wang ${ }^{\star, \#}$ \\ Institute of Microstructure and Property of Advanced Materials, Beijing University of \\ Technology, 100022 Beijing, China, Key Laboratory of Coal Conversion, Institute of \\ Coal Chemistry, Chinese Academy of Sciences, Taiyuan 030001, People's Republic of \\ China, Materials Science Department, Tsinghua University, 100084, Beijing, China, \\ and School of Materials Science and Engineering, Georgia Institute of Technology, \\ Atlanta, Georgia 30332
}

Received November 27, 2006; Revised Manuscript Received December 12, 2006

\begin{abstract}
Large strain plasticity is phenomenologically defined as the ability of a material to exhibit an exceptionally large deformation rate during mechanical deformation. It is a property that is well established for metals and alloys but is rarely observed for ceramic materials especially at low temperature $(\sim 300 \mathrm{~K})$. With the reduction in dimensionality, however, unusual mechanical properties are shown by ceramic nanomaterials. In this Letter, we demonstrated unusually large strain plasticity of ceramic SiC nanowires (NWs) at temperatures close to room temperature that was directly observed in situ by a novel high-resolution transmission electron microscopy technique. The continuous plasticity of the SiC NWs is accompanied by a process of increased dislocation density at an early stage, followed by an obvious lattice distortion, and finally reaches an entire structure amorphization at the most strained region of the NW. These unusual phenomena for the SiC NWs are fundamentally important for understanding the nanoscale fracture and strain-induced band structure variation for high-temperature semiconductors. Our result may also provide useful information for further studying of nanoscale elastic-plastic and brittle-ductile transitions of ceramic materials with superplasticity.
\end{abstract}

Large strain plasticity is phenomenologically defined as the ability of a material to exhibit exceptionally large deformation rate during mechanical deformation. ${ }^{1}$ It is a property of some polycrystalline solids ${ }^{2}$ and has been well established for metals and alloys. ${ }^{3}$ With the reduction in dimensionality, nanomaterials exhibit unusual and unique behavior such as high hardness and high strength that have been demonstrated in nanowires of metals ${ }^{4}$ and ionic ${ }^{5}$ and covalent ${ }^{6}$ materials. For ceramic materials, it is naturally hard but very brittle. Large strain plasticity of bulk ceramic materials does not occur at low temperatures. Ceramics are brittle materials at room temperature and they usually break at small strains of $0.1-0.2 \%$ or even smaller. ${ }^{7}$ Bulk brittle materials can transform to ductile by creating dislocation and fostering dislocation motion at high temperatures, even as high as $2 / 3$

* Corresponding authors: zezhang@bjut.edu.cn (Z.Z.) and zhong.wang@ mse.gatech.edu (Z.L.W.).

$\dagger$ Institute of Microstructure and Property of Advanced Materials, Beijing University of Technology.

$\doteqdot$ Key Laboratory of Coal Conversion, Institute of Coal Chemistry, Chinese Academy of Sciences.

$\S$ Materials Science Department, Tsinghua University.

\# School of Materials Science and Engineering, Georgia Institute of Technology. of the melting point $T_{\mathrm{m}}$ of the bulk ${ }^{8}$ or phase transformation at high pressure. Understanding the atomic mechanisms and dynamics of a brittle material impacted by an external force is fundamentally important to theoretical and applied physics, such as atomic lattice elastic-plastic response $\mathrm{e}^{9,10}$ brittleductile (B-D) transition, ${ }^{11-13}$ materials toughness, hardness, ${ }^{14}$ and fractures. ${ }^{15}$ Recently, with the emergence of new directions in flexible dimensionality of electronic devices and forms ${ }^{16,17}$ as well as advancements in single nanowire (NW) electronics, ${ }^{18}$ it has become crucial to assess the nanoscale mechanical responses such as elastic-plastic deformation of the most important semiconductor materials, such as $\mathrm{Si}$ and $\mathrm{SiC}$.

Experimentally, nanoindentation, ${ }^{19,20}$ atomic force microscopy (AFM), ${ }^{4,6}$ and in situ transmission electron microscopy $(\mathrm{TEM})^{21,22}$ have been used to characterize the nanomechanical properties of nanodomains ${ }^{19,20}$ and single NWs. ${ }^{4,22}$ From the theoretical perspective, recent developments in simulation techniques have opened new approaches for investigating the microscopic origins of complex nanomaterial phenomena. ${ }^{10,19,23,24}$ However, contradictive results have emerged; for example, brittle-fracture features with small strain were 


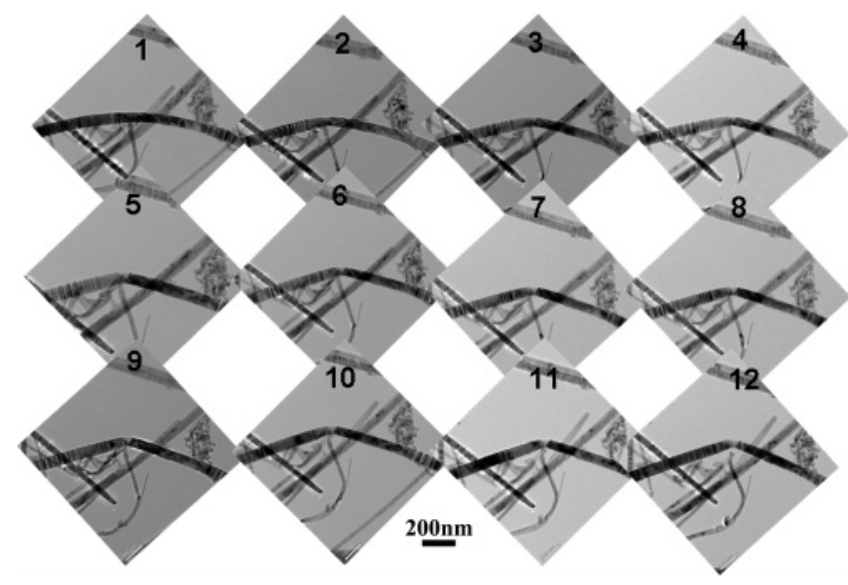

Figure 1. In situ TEM observation of the plastic deformation features of a SiC NW under large angle bending. The plastic deformation was triggered from the second image. The time interval between image 1 and 2 was about $30 \mathrm{~min}$, and the entire procedure lasted about $3 \mathrm{~h}$.

observed by large atomic number molecular dynamics (MD) simulations ${ }^{23,36}$ for $\mathrm{SiC}$ NWs. On a contrary scenario, large strain elasticity and ductile fracture features were achieved. ${ }^{25}$ Obviously, experimental evidence is necessary and mandatory to clarify the true deformation features and mechanisms of the ceramic nanowires. A full understanding about the atomic scale features of a NW under deformation remains a challenge due to the difficulty of simultaneous testing and nanoscale imaging. In particular, with respect to the elasticplastic and $\mathrm{B}-\mathrm{D}$ transitions, the physical picture regarding the atomic scale mechanisms and dynamics of an individual NW remains to be investigated.

In this Letter, we use the recently developed in situ TEM technique (see Supporting Information and Figure S1) to perform bending deformation of single SiC NWs. We present complete and dynamic atomic evolution images to reveal the very unusual mechanical behavior of a SiC NW under deformation. The observation may also help us to understand some of the intrinsic and/or universal mechanical behavior of nanoscale materials of semiconducting or ceramic characteristic. The in situ SiC NW elastic, elastic-plastic and plastic deformations were conducted in an ultrahigh resolution transmission electron microscope by bending SiC NWs via the mechanical force produced by the transmission electron microscope specimen-supporting grid under the irradiation of the electron beam ${ }^{26,27}$ (see Supporting Information). Figure 1 shows a series of images of a single SiC NW that was being deformed by the force created from the TEM grid under the irradiation/heating of an electron beam. A clear plastic deformation is observed at the bending region. Instead of a crystalline fracture, the NW has transformed from crystalline to amorphous at the mostly strained region. Among of $20 \mathrm{SiC}$ NWs that had been studied, all of them had shown large elastic strain (up to $2 \%$ ), and four of them revealed plastic deformation with the strain aggressively extended. Parts a and c of Figure 2 show an example of the starting elastic deformation of a SiC NW and parts b and d of Figure 2 display the final plastically deformed images of the single bent $\mathrm{SiC} \mathrm{NW}$, respectively. The series of images
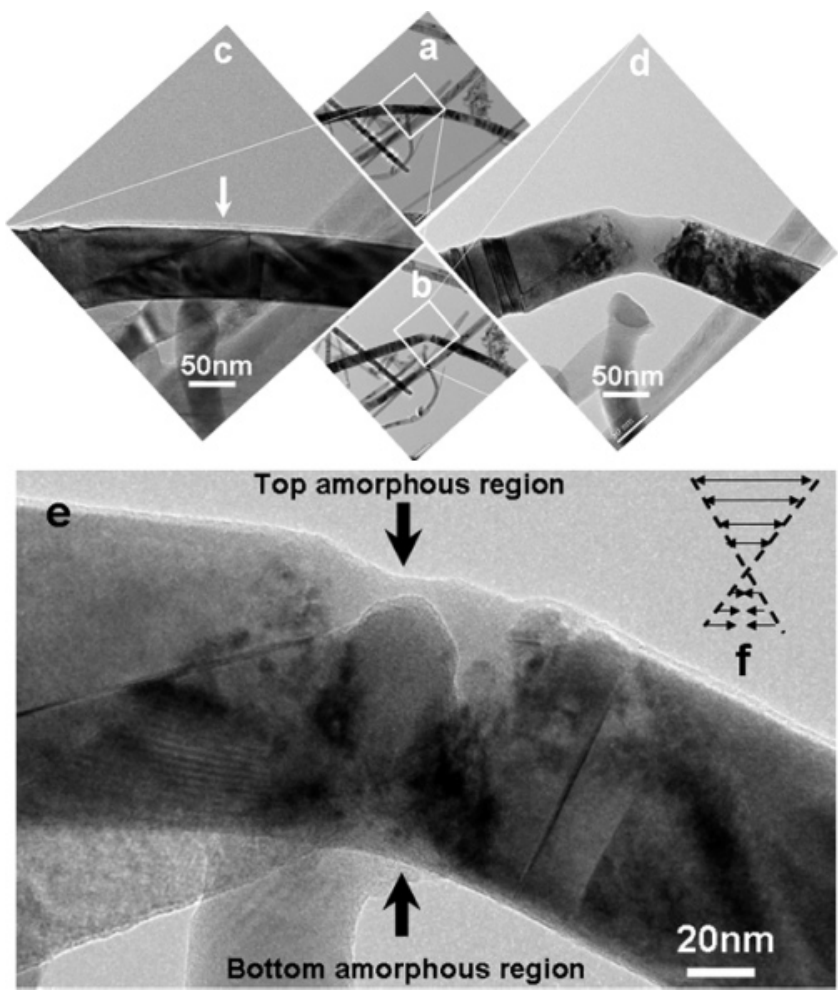

Figure 2. SiC NW morphologies of the elastic bend (a, c) and the final plastic bend of (b) and (d). The location indicated by the white arrow in (c) is the triggering point of plastic deformation. (e) is an enlarged TEM bright field image. The black arrows in (e) show the top and bottom deformation-induced amorphous zones. The asymmetric amorphous zone perfectly reflects the butterfly-shaped or X-shaped strain field as shown in (e) and (f).

captured in situ in both the elastic and plastic deformation modes can be viewed in Figure 1. The strain rate was kept at a level of $10^{-4}-10^{-5} / \mathrm{s}$. The up limit strain for inducing elastic deformation of $\mathrm{SiC} \mathrm{NW}$ at relatively low temperature is around $2 \%$, which is within the same order of magnitude as that reported for ex situ studies, ${ }^{28}$ and is consistent with theoretical predictions. ${ }^{29}$ For the in situ observed plastically bent $\mathrm{SiC} N W$, until the diameter of the plastic necking zone decreased from 86 to $67 \mathrm{~nm}$, a large plastic strain at the plastic necking valley has been achieved. We could not observe any cracks even at nanoscale or atomic scale. Accordingly, we can predict that the fracture strain of $\mathrm{SiC}$ NWs with a diameter of tens of nanometers could be as high as few hundreds percent or even more, which reveals a large strain plasticity nature. To our knowledge, this is the first observation of large strain plasticity of SiC NWs at temperatures close to room temperature.

One of the key questions that one may ask is how high is the local temperature under the electron beam. Our experiment was carried out at $200 \mathrm{kV}$ and with a low electron flux dose rate around $5 \times 10^{19} \mathrm{e} /\left(\mathrm{cm}^{2} \mathrm{~s}\right)$. According to the model presented by Jencic et al. (ref S3), a simple calculation for such a dosage indicates that the local temperature is no more than a few degrees above room temperature. More importantly, during the entire experiments, the electron beam was spread to illuminate an area that is as large as $10-20$ $\mu \mathrm{m}$ and we avoided the full convergence of the beam to a 


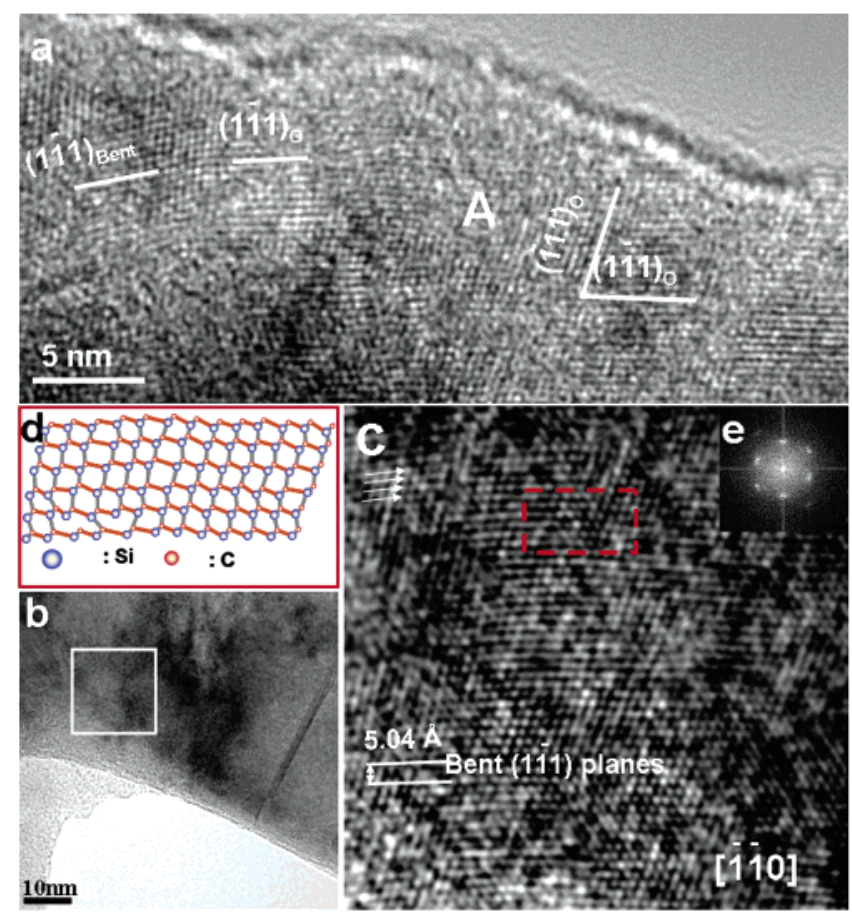

Figure 3. (a) The topmost regions, revealing the well-developed plastic-deformation features. The subscript "O" represents original lattice while "bent" means the bent lattices. (b) A low-magnification image of the bent SiC NW from the compressive side. (c) The enlarged area taken from the framed region of (b) to show the incipient elastic-plastic transition features at the atomic scale. (d) The head-tail connection of the $\mathrm{Si}-\mathrm{C}$ atom pairs ${ }^{30}$ to represent the two-dimensional projection of the $\mathrm{Si}-\mathrm{C}$ atomic pairs. It shows more clearly the characters of the $(1 \overline{1} 1)$ bent lattices and dislocations/stacking faults on the (111) planes. The blue open circle indicates the $\mathrm{Si}$ atoms and the orange open circle represents the carbon atoms. The gray bars illustrate the bonds on the $\{111\}$ shuffle plane and the orange ones are those for the $\{111\}$ glide planes. See Figure 4 for more details.

small area to eliminate radiation damage. Therefore, the temperature effect and irradiation damage introduced by the electron beam were negligible.

The starting amorphous regions possess typical bendinginduced tensile-compressive features with up-tensile, bottom compressive, and center-neutral stress areas. As shown in parts e and $f$ of Figure 2, an astonishing phenomenon is that a butterfly shaped tensile-compressive amorphous zone is clearly visible and reveals the asymmetric features of the tensile-compressive strain field. The top and bottom regions are the highest level strain regions and therefore are the first to trigger plastic deformation. The plastic deformation is well visualized by the formation of an amorphous phase with distinctive contrast compared to the crystalline regions. ${ }^{30}$ The central tipped object with gray contrast shown in Figure 2e comes from a NW underneath the one that was being bent. It drifted away from the examined NW in the later bending process, as shown in parts $b$ and $d$ of Figure 2. The neutral stress zone shifted to the compressive side as indicated in Figure 2e. The shift of the neutral stress zone can be understood by the fact that the plastic deformation happened on the convex of the NW first due to asymmetrical features of the Schmidt factors under tensile and compressive strain loads $^{31}$ and then shifted toward the concave direction.
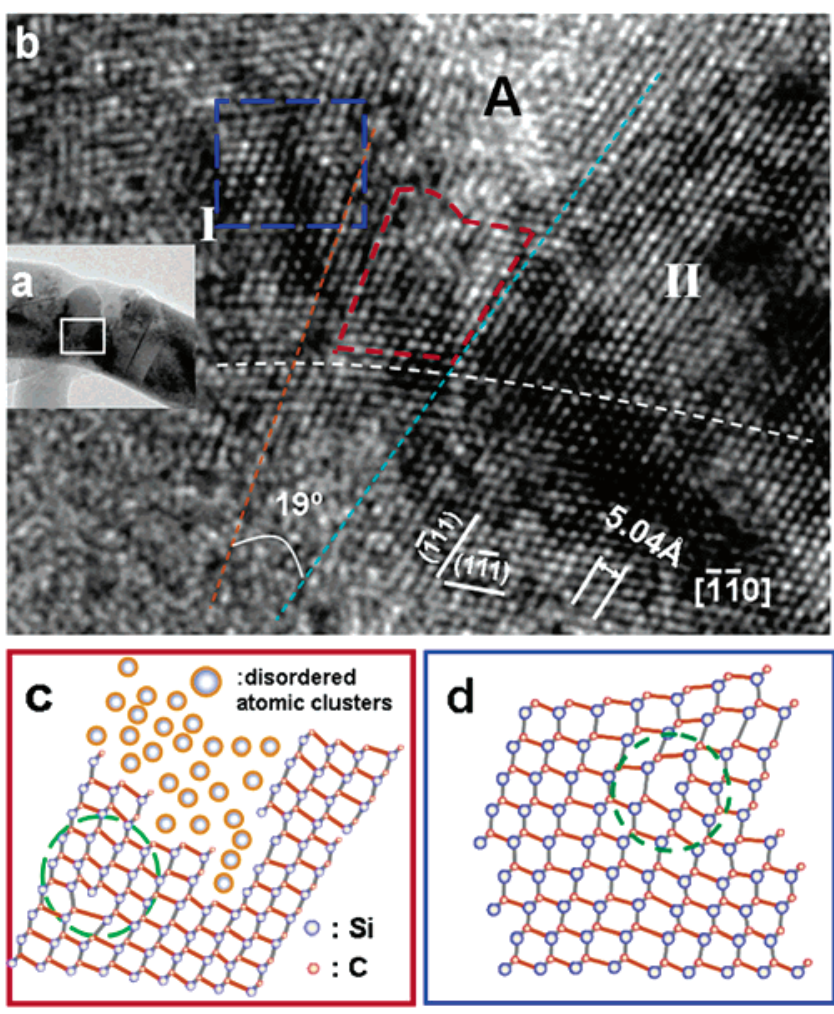

Figure 4. An area at the middle stage of plastic bending of the bent SiC NW showing the features of lattice bending, formation of dislocations, and the process of amorphization. (a) A low magnification image. (b) The enlarged area of the framed region of (a). Two areas are picked up, and we use the classic head-tail connection $^{30}$ to represent the two-dimensional projection of the $\mathrm{Si}-\mathrm{C}$ atomic pairs. The symbols have identical meanings with those of Figure 3. The white dashed line indicates the bent lattice from region II to I, and the bent magnitude is represented by a $19^{\circ}$ angle between the orange and teal lines. Part $\mathrm{c}$ is a drawing of the redframed area in (b) and part $\mathrm{d}$ is a drawing showing the features for the blue-framed region in (b). The green circled regions in (c) and (d) indicate dislocated areas around the tip of the "crack-shaped" amorphous valley. (c) reveals a dislocation on the (111) plane and (d) contains a dislocation on the (111) plane. See text for details.

To shed light on the atomic mechanism about the elasticplastic and pure plastic deformation in the SiC NWs, a close view of the deformation process is conducted. At the atomic level, as revealed in Figure 3a, the top plastic deformed areas are a mixture of disordered atomic lattices/planes, bent lattices, and amorphous zones. This is similar to our previous reported case in an ex situ experiment at a higher strained rate. ${ }^{28}$ In fact, the morphology shown in Figure 3 a occurs at the near-end of the plastic-deformed amorphization process rather than at the beginning. Parts $\mathrm{b}$ and $\mathrm{c}$ of Figure 3 show an early incipient stage of the elastic-plastic transition that is characterized by homogeneous lattice distortion, dislocation nucleation, and lattice relaxation in the compressed side of the bent $\mathrm{SiC}$ NW. Figure $3 \mathrm{c}$ is an enlarged high-resolution electron microscopy (HREM) image taken from the framed region of Figure 3b. The elastic/plastic anisotropy is revealed in the incipient elastic-plastic transition period in the bent $\mathrm{SiC}$ NW when an external force is applied. The periodicity and continuity of the $\{111\}$ family of lattice planes have 

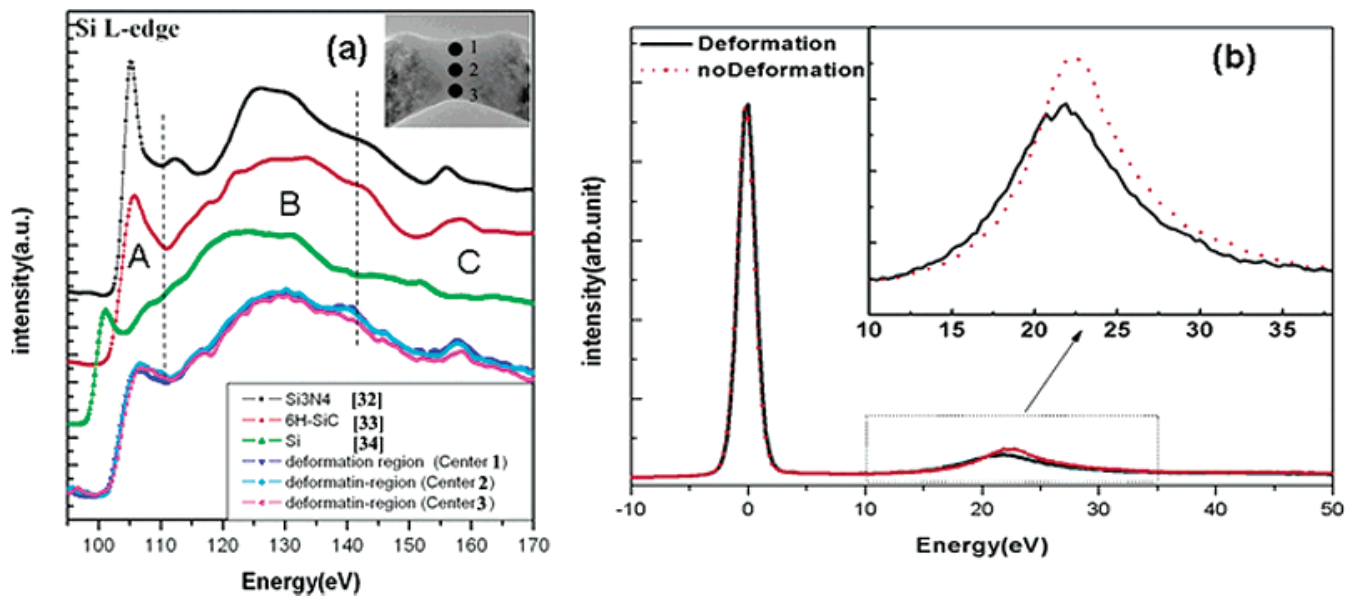

Figure 5. The EELS spectra taken from the deformed regions of the SiC NW. For the reason of comparison, some reference data are also shown in the same figure. See text for details.

been investigated. Neglecting the exact silicon and carbon atomic coordinates, we may assume that the white dots in the high-resolution TEM images are the projections of $\mathrm{Si}-\mathrm{C}$ atomic "dumbbell" pairs, ${ }^{30}$ as in conventional lattice images. Figure $3 \mathrm{~d}$ is plotted based on the red-framed region in the HREM image shown in Figure 3c. Figure 3e is a fast Fourier transformation (FFT) pattern corresponding to Figure $3 \mathrm{c}$ to show the lattice bending features which are reflected in the "arc" characteristic of the diffraction spot in reciprocal space. It is interesting to note that the continuity of the atoms on the trace of (11) plane is conserved better than that of (111) planes. This indicates that the atomic slip occurs by gliding

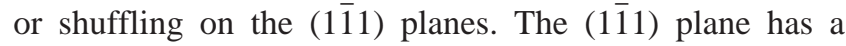
favorable Schmidt factor with respect to the direction of the applied external force. The incipient elastic-plastic transition anisotropy in the bent SiC NW derives from the distinguishable Schmidt factor values of the potential active slip systems of $\{111\}\langle 110\rangle$ with respect to the applied external force. This phenomenon indicates that the Schmidt law is applicable at the nanoscale. The initiation of elastic-plastic transition by dislocation nucleation is consistent with a very recent nanoindentation simulation on nanocrystalline $\mathrm{SiC}$ thin film. ${ }^{24}$ However, no large strain plastic deformation could be conducted and no unusual low-temperature superplasticity was predicted in ref 24 .

The HREM images recorded later in the process reveal the atomic mechanisms of the plastic deformation features with large strains. Figure 4a shows a low magnification image at a later stage of the "tearing/fracturing" process and (b) is a HREM image corresponding to the boxed area in (a). Figure $4 \mathrm{~b}$ shows the up-central, fully developed amorphous region indicated by " $\mathrm{A}$ " and the two tearing apart areas that are labeled with I on the left-hand side and II on the right-hand side of the fracture tip. Selecting two regions near the amorphous zone in the HREM image of Figure $4 \mathrm{~b}$ and conducting the classical head-tail connection of $\mathrm{Si}-\mathrm{C}$ atomic pairs by tracing the white dots (projected $\mathrm{Si}-\mathrm{C}$ atomic pairs), the two-dimensional atomic projections along the [11 10$]$ direction are shown in parts $\mathrm{c}$ and $\mathrm{d}$ of Figure 4. The crystalline lattice in areas I and II has been bent/torn as indicated by the white dashed line. The size of the bending is $19^{\circ}$. A dislocation lying in the (111) plane in the red frame area is inserted to compensate for the large angle bending. In the HREM image shown in Figure 4b, a close inspection reveals that the tip of the "fracture" is blunted by surrounding dislocations, which is consistent with theoretical predictions. ${ }^{13}$ Figure $4 \mathrm{c}$, which is drawn to represent the red-framed region in Figure $4 \mathrm{~b}$, shows the dislocation forming on the (111) plane with a Burgers vector along [211] (or the projection of the [110] direction). Dislocations sited in a different set of close-packing atomic planes have also been observed. Figure $4 d$, which is drawn to represent the blueframed area of Figure $4 b$, shows the dislocations on the (1) 1 ) plane with Burgers vector along the [121] (also possible is a projection of $[10 \overline{1}])$ direction. The amorphous region propagates through dislocations, atomic lattice disordering, and relaxation.

Further study of the SiC NWs by in situ electron energy loss spectroscopy (EELS) offers additional evidence about our study on the mechanism of plastic deformation. Figure 5 shows the in situ EELS analysis results on the bent $\mathrm{SiC}$ NW. Figure 5a is the EELS results of the Si-L edge taken from three centered points of the bent $\mathrm{SiC}$ NW. Here we only show the information of $\mathrm{Si}$ because the structure of carbon may not be intrinsic due to the possible carbon contamination in TEM. For the reason of comparison, we illustrate the structure of $\mathrm{Si}-\mathrm{L}$ edge of $\mathrm{Si}_{3} \mathrm{~N}_{4},{ }^{32} 6 \mathrm{H}-\mathrm{SiC}, 3 \mathrm{C}$ $\mathrm{SiC}^{33}$ and $\mathrm{Si}^{34}$ in the same figure. The inset in Figure $5 \mathrm{a}$ indicates the locations of labeled 1, 2, and 3 from where the EELS spectra of the bent SiC NW were recorded. Comparing to the $\mathrm{sp}^{3}$ bonding structures of $\mathrm{Si}_{3} \mathrm{~N}_{4}$ and the crystalline $6 \mathrm{H}-$ $\mathrm{SiC}$, the peak in the spectra from region $\mathrm{A}$ of the deformed $\mathrm{SiC}$ expands to a wider range with amorphous features. In contrast to the structure of the electron-irradiated $\mathrm{SiC},{ }^{35}$ we conclude that the peak at region $\mathrm{A}$ is the typical feature of the amorphous phase. However, the fine structures of the deformed $\mathrm{SiC}$ shown in region $\mathrm{B}$ and region $\mathrm{C}$ have similar structures to those of crystalline structure of $6 \mathrm{H}-\mathrm{SiC}$ and 


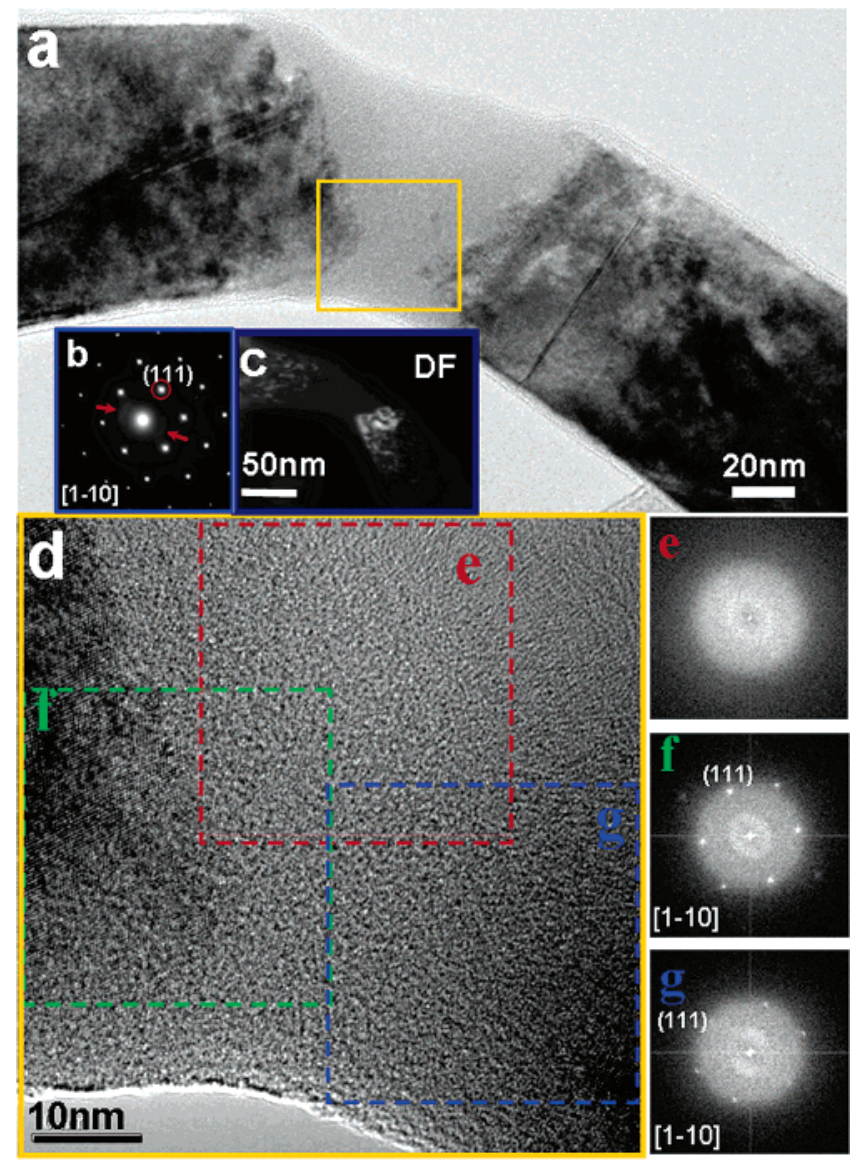

Figure 6. The amorphous structure at the necked valley of the plastically deformed $\mathrm{SiC} \mathrm{NW}$. (a) A bright field low-magnification HREM image to show the features of the plastically deformed SiC NW. (b) A selected area electron diffraction pattern taken from both of the amorphous and crystalline regions. (c) A dark field image obtained by selecting the (111) diffraction spot of (b). The bright contrast regions in (c) are the crystalline areas which contribute to the diffraction of (111). The central dark regions are the plastically deformed amorphous areas which do not participate in the (111) crystalline plane diffraction. (d) A high-magnification HREM image taken from the framed region of (a). The three selected regions are marked by red, green, and blue frames and conducted fast Fourier transformation (FFT) as shown in (e), (f), and $(\mathrm{g})$, respectively.

$\mathrm{Si}_{3} \mathrm{~N}_{4}$. The characters of the EELS peaks at zone $\mathrm{C}$ of the deformed $\mathrm{SiC}$ at the peak position $\mathrm{P}$ shift to a high-energy position for about $3-5 \mathrm{eV}$. To confirm the amorphous features of the deformed $\mathrm{SiC}$, we studied the plasma peak of the bent $\mathrm{SiC}$ as shown in Figure 5b. The plasma peak of the bent SiC NW shifts to the higher energy compared to the undeformed one, and this is a character of the disordered structure. In addition to the EELS analysis, to unambiguously demonstrate the amorphous structure of the plastically deformed $\mathrm{SiC} \mathrm{NW}$, we studied the plastically deformed necking-valley region of the bent $\mathrm{SiC} \mathrm{NW}$ by selected area electron diffraction and dark-field imaging as well as highmagnification HREM imaging. Figure 6a is a low-magnification bright field HREM image to show the features of the plastically deformed $\mathrm{SiC} \mathrm{NW}$. In the selected area diffraction pattern as shown in Figure 6b, the small red arrows indicate the halo ring segments, which are typical feature of amor- phous. Figure $6 \mathrm{c}$ is the dark field image by selecting the (111) diffraction spot of Figure $6 \mathrm{~b}$ which is indicated by a small red circle. The bright contrast regions in Figure $6 \mathrm{c}$ are the crystalline areas that contribute to the diffraction of (111). The central dark regions are the amorphous areas which do not participate in the (111) crystalline plane diffraction. Figure $6 \mathrm{~d}$ is a high-magnification HREM image taken from the framed region of Figure 6a. Three regions were selected to conduct the FFT as shown in parts $\mathrm{e}-\mathrm{g}$ of Figure 6, respectively. Parts $f$ and $g$ of Figure 6 show crystalline features, while Figure 6e has typical amorphous character. Some crystalline lattice features can be revealed in the left bottom area (f) of Figure $6 \mathrm{c}$ and some crystalline domains/ islands can be eventually found in the amorphous "sea" in the enlarged HREM image of Figure 6c. On the basis of the above analysis, we suggest that the amorphous-like deformation zone of $\mathrm{SiC}$ contains both disordered and tiny crystalline units. The $\mathrm{sp}^{3}$ bonding features were kept in the deformed $\mathrm{SiC}$ regions. The structure of the necked "amorphization zone" of the deformed $\mathrm{SiC}$ has distinctive fine structure compared to the electron-irradiation induced amorphous $\mathrm{SiC}$.

It is worthwhile to point out that the strength of the $\mathrm{SiC}$ $\mathrm{NWs}^{6}$ is the highest one so far among all of the available NWs having ever been tested. It is close to the theoretical predicted value of $5 \% \mathrm{E} .{ }^{37}$ But the data reported in ref 6 has reached a value that is twice that of the theoretical limit by Orowan expression estimation. ${ }^{37}$ Our observation may offer a reasonable interpretation about this result. The plastic deformation process could be involved in the bending case of $\mathrm{SiC}$ NWs in ref 6. Derived from present observation, we suggest that metal-like dislocation interactions, lattice disordering, and amorphization occurred simultaneously in the lateral bending process reported in ref 6 . The conservation of $\mathrm{sp}^{3}$ bonding in the amorphous phase contributes to the continuous increase of the bending strength of SiC NWs. A three-step deformation was involved in this process in which a strain-induced phase transition from crystalline to amorphous was observed in this study.

The results clearly revealed the distinct mechanical properties of individual $\mathrm{SiC}$ NWs with a bending-induced large strain plasticity at temperatures close to room temperature, which has never been observed in bulk ceramic materials. The large strain plasticity may correspond to superstrength. The direct dynamic atomic level elasticplastic and $\mathrm{B}-\mathrm{D}$ transition images and mechanisms for a single SiC NW are different from those of the bulk materials and amplify the strong scale effect. The process is distinctive from the "one step transition" mechanism in the MD simulation from the crystalline to the amorphous phase..$^{23,25,36}$ It is also different from another type of thermal/stressactivated elastic instability and $\mathrm{B}-\mathrm{D}$ transition in which the mobility of the existing dislocations is the main concern. ${ }^{11}$ The scale effect contributes to the new triple-step deformation mechanisms of the ceramic NWs in addition to the wellaccepted temperature and strain rate factors. The unusual phenomena found for the $\mathrm{SiC}$ NWs are fundamentally important for understanding the strain-induced band structure variation and the corresponding changes in transportation 
property of semiconductor NWs. Our result may also provide useful information for further studying of nanoscale elasticplastic and brittle-ductile transformation of ceramic materials with superplasticity.

Acknowledgment. This work was supported by China National 973 program under No. 2002CD613500 and National Science Foundation of China. Xiaodong Han is grateful for valuable discussions with Professor K. H. Kuo who was formerly with the Beijing Laboratory of Electron Microscopy (BLEM), Chinese Academy of Sciences. This research is supported by Funding Project for Academic Human Resources Development in Institutions of Higher Learning under the Jurisdiction of Beijing Municipality. The authors thank Drs. Rhoda E. and Edmund F. Perozzi, of the Beijing University of Technology, for extensive editing assistance. Z.L.W. thanks the support from the US NSF, DARPA and NIH.

Supporting Information Available: Experimental details providing general information, electron beam temperature increase, and calculation of elastic strain. This material is available free of charge via the Internet at http://pubs.acs.org.

\section{References}

(1) Edington, J. W.; Melton, K. N.; Cutler, C. P. Prog. Mater. Sci. 1976, 21,61 .

(2) Lu, L.; Sui, M.; Lu, K. Science 2000, 287, 1463.

(3) Bricknell, R. H.; Edington, J. W. Acta Metall. 1979, 27, 1313.

(4) Wu, B.; Heidelberg, A.; Boland, J. J. Nat. Mater. 2005, 4, 525.

(5) Chen, C. Q.; Shi, Y.; Zhang, Y. S.; Zhu, J.; Yan, Y. J. Phys. Rev. Lett. 2006, 96, 075505.

(6) Wong, E. W.; Sheehan, P. E.; Lieber, C. M. Science 1997, 277, 1971.

(7) Green, D. J. An Introduction to the Mechanical Properties of Ceramics; Cambridge University Press: Cambridge, 1998.

(8) Hirsch, P. B.; Roberts, S. G. Acta Mater. 1996, 44, 2361.

(9) Born, M.; Huang, K. Dynamic Theory of Crystal Lattices; Oxford University Press: Oxford, 1954.

(10) Li, J.; Vliet, J. K.V.; Zhu, T.; Yip, S.; Suresh, S. Nature 2002, 418, 307.

(11) Gumbsch, P.; Riedle, J.; Hartmaier, A.; Fischmeister, H. F. Science 1998, 282, 1293.
(12) Hirsch, P. B.; Roberts, S. G.; Samuels, J. Proc. R. Soc. London, Ser. A 1989, 421, 25.

(13) Cheung, K. S.; Yip, S. Phys. Rev. Lett. 1990, 65, 2804.

(14) Gao, F.; He, J.; Wu, E.; Liu, S.; Yu, D.; Li, D.; Zhang, S.; Tian, Y. Phys. Rev. Lett. 2003, 91, 015502.

(15) Buehler, M. J.; Gao, H. Nature 2006, 439, 307.

(16) Jacobs, H. O.; Tao, A. R.; Schwartz, A.; Garcias, D. H.; Whitesides, G. M. Science 2002, 296, 323.

(17) Khang, D. Y.; Jiang, H. Q.; Huang, Y.; Rogers, J. A. Science 2006, $311,208$.

(18) Huang, Y.; Duan, X. F.; Cui, Y.; Lauhon, L. J.; Kim, K.; Lieber, C. M. Science 2001, 294, 1313.

(19) Landman, U.; Luedtke, W. D.; Brunham, N. A.; Colton, R. J. Science 1990, 248, 454.

(20) Li, X. D.; Gao, H. S.; Murphy, C. J.; Caswell, K. K. Nano Lett. 2003, 3, 1495.

(21) Poncharal, P.; Wang, Z. L.; Ugarte, D.; Heer, W. A. Science 1999, $283,1513$.

(22) Huang, J. Y.; Chen, S.; Wang, Z. Q.; Kempa, K.; Wang, Y. M.; Jo, S. H.; Chen, G.; Dresselhaus, M. S.; Ren, Z. F. Nature 2006, 439, 281.

(23) Li, W.; Kalia, R. K.; Vashishta, P. Phys. Rev. Lett. 1996, 77, 2241.

(24) Szlufarska, I.; Nakano, A.; Vashishta, P. Science 2005, 309, 911.

(25) Kim, T. Y.; Han, S. S.; Lee, H. M. Mater. Trans. 2004, 45, 1442.

(26) Chinese Patent: Application Number: 200610057989.5

(27) Supporting Information.

(28) Han, X. D.; Zhang, Y. F.;Liu, X. Q.; Zhang, Z.; Hao, Y. J.; Guo, X. Y. J. Appl. Phys. 2005, 98, 124307.

(29) Wang, J. H.; Yip, S.; Phillpot, S. R.; Wolf, D. Phys. Rev. Lett. 1993, $71,4182$.

(30) Cowley, J. M. Diffraction Physics; North-Holland Publishing Company: New York, 1975.

(31) Gall, K.; Sehitoglu, H.; Chumlyakov, Y. I.; Kireeva, I. V. Acta Mater. 1999, 47, 1203.

(32) Batson, P. E.; Kavanagh, K. L.; Wong, C. Y.; Woodall, J. M. Ultramicroscopy 1987, 22, 89 .

(33) Skiff, W. M.; Carpenter, R. W.; Lin, S. H. J. Appl. Phys. 1985, 58, 3463.

(34) Muto, S.; Tanabe, T. J. Appl. Phys. 2003, 93, 3765.

(35) Ishimaru, M.; Bae, I.; Hirata, A.; Hirotsu, Y. Phys. Rev. B 2005, 72, 024116.

(36) Tanaka, H.; Sano, M.; Shimada, S. Key Eng. Mater. 2004, 257$258,15$.

(37) Lambrecht, W. R. L.; Segall, B.; Methfessel, M.; Schilfgaarde, M. V. Phys Rev. B 1991, 44, 3685.

NL0627689 